\title{
Investigating the Multibody Dynamics of the Complete Powertrain System
}

\author{
Alessandro Picarelli \\ Mike Dempsey \\ Claytex Services Ltd. \\ Edmund House, Rugby Road, Leamington Spa, CV32 6EL, UK \\ alessandro.picarelli@claytex.com mike.dempsey@claytex.com
}

\begin{abstract}
The specifications and integration of two new Modelica libraries is presented: The Powertrain Dynamics (PTDynamics) and the Engines libraries. The libraries enable the simulation and modelling of powertrain systems including their fluid dynamic, pollutant emission, mechanical and thermal performances in one simulation environment (Dymola), utilising the object orientated modelling language Modelica.

Two variants of the Engines library are presented: a Mean Value variant (MVEL) and a Crank Angle Resolved variant (CAREL).

Both the PTDynamics and Engines libraries make use of a new approach to modelling the mechanics that captures the full MultiBody effects of the Powertrain system without the computational cost of using the standard Modelica MultiBody library.
\end{abstract}

\section{Introduction}

Development of powertrain systems and components is an ongoing and relentless activity in the automotive industry. The ability to reduce the number of expensive prototypes, engineering costs and development time is an attractive feature of CAE simulation tools.

Simulation time performance is an important feature particularly in real-time applications such as for SIL (Software-in-the-loop) and HIL (Hardware-in-theloop) experiments. For this particular reason, the libraries presented in this paper have been designed with efficiency in mind and show reduced model complexity when compared to analogous system models built with the Modelica standard library components whilst retaining equal or improved levels of accuracy.

\section{Engine Library}

The Engine library is capable of modelling both Spark Ignition (SI) and Compression Ignition engines and is split into two variants with different levels of fidelity. Both levels of the Engines library have been designed to work with common engine architecture templates. This enables quick model setup and ensures a consistent layout for a variety of engine architectures.

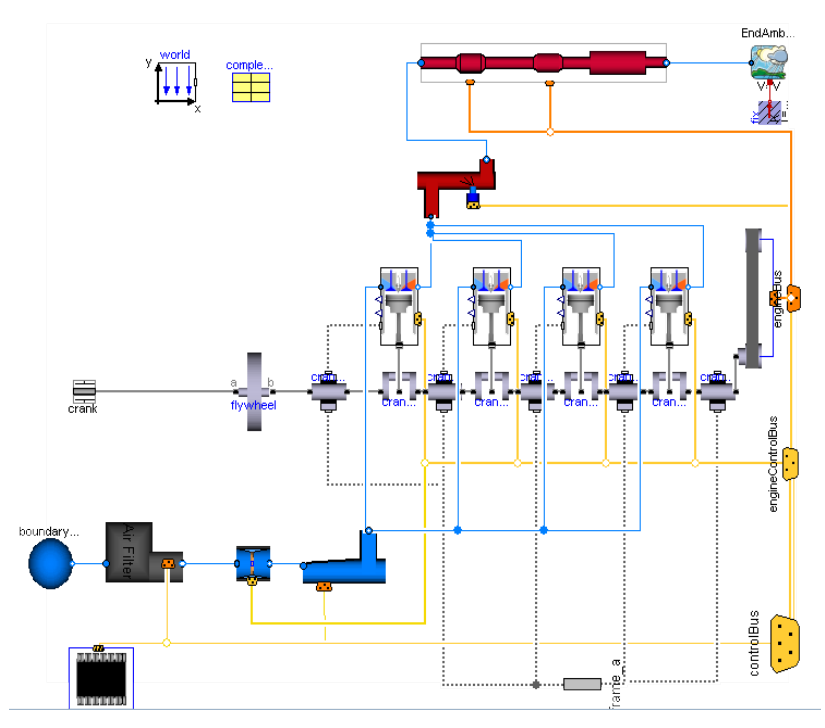

Example of a multi-cylinder MVEM layout

The mechanical components are modelled using a new Rotational3D library described in section 3 and the Fluids models are based on the new Modelica Fluids library [1].

\subsection{Mean Value Engine Library (MVEL)}

This level of the library is capable of predicting cycle-averaged values for engine torque, thermal effects and emissions. The methods for predicting the engine torque and emissions are map based and/or neural network based. 
The library is of particular use for investigating different control algorithms and their effect on the engine transient response. This variant of the engine library is also suited to driveability analysis where the transient torque output of the engine is fed through the transmission and reacted into the transmission and engine mounts. Furthermore, engine models using this library are capable of running in real time making it suitable for SIL and HIL testing of control systems.

Mass flow rate through the engine cylinders is computed by means of [2]:

Where:

$$
\dot{m}_{a p}\left(n, p_{i}\right)=\frac{V_{d}}{120 R T}\left(s_{i} p_{i}+y_{i}\right) \frac{n}{1000}
$$

$\mathrm{m}$ is the mass of fluid within the cylinder, $V_{d}$ is the volumetric displacement of each cylinder

$R$ is the gas constant

$T$ is the fluid temperature $(\mathrm{K})$

$p_{i}$ is the intake manifold pressure (bar)

$n$ is the engine speed in rpm.

The above equation relates the mass flow rate through each cylinder to the engine speed and intake manifold pressure. The equation can be scaled to account for variations in engine displacement provided the engine technology and valve timing remains similar.

Derivation of the engine specific constants $s_{i}$ and $y_{i}$ must be arranged prior to use. These are obtained by a linear fit of a rearranged version of the mass flow rate function (shown below) vs. intake manifold pressure.

$$
\dot{m}_{a p} * \frac{120 R T^{*} 1000}{V_{d} * n}=\left(s_{i} p_{i}+y_{i}\right)
$$

The linear fit yields an equation in the form of $y=$ $\mathrm{mx}+\mathrm{c}$, where the gradient $\mathrm{m}$ will be equal to $\mathrm{s}_{\mathrm{i}}$ and the intercept $\mathrm{c}$ will be equal to $\mathrm{y}_{\mathrm{i}}$ :

If the engine makes use of variable induction mechanisms (variable valve timing, variable length intake runners, etc.) the user might wish to determine $s_{i}$ and $y_{i}$ for various engine speed intervals, to improve the accuracy of the mass flow rate. A map of these values can then be input into the model.

Once $\mathrm{s}_{\mathrm{i}}$ and $\mathrm{y}_{\mathrm{i}}$ have been determined, the computed mass flow rate is used in a mass flow rate source and sink, each representing the flow past the intake valves and the exhaust valves respectively.

The intake and exhaust system mediums are based on Modelica.Media medium models and are modelled as separate fluids, each containing the appropriate species for that part of the engine.
The main reason for utilising two specific medium models (intake and exhaust), as opposed to a single medium model encompassing all intake and exhaust species, is down to CPU time reduction.

Whole engine medium:

Species 1

Species 2

Species 3

Species 4

Species 5

Species 6

Species 7

\begin{tabular}{c:c} 
& \multicolumn{1}{c}{} \\
Intake medium: & Exhaust Medium: \\
Species 1 & Species 4 \\
Species 2 & Species 5 \\
Species 3 & Species 6 \\
& Species 7
\end{tabular}

If we were to use the whole engine medium model, we would have to:

- set the exhaust specific species mass fractions to zero on the intake side

- $\quad$ set the intake specific species mass fractions to zero on the exhaust side

Due to computational errors the mass fractions of these mediums might end up not being exactly equal to zero. By using two separate, simpler, medium models the mass fraction balancing becomes more robust and gains in CPU time are achieved.

\subsection{Crank Angle Resolved Engine Library (CAREL)}

This variant of the engine library is capable of predicting crank angle resolved values for torque, thermal effects and friction with more detailed intake, exhaust and combustion models. Typical applications of this variant of the library would be for investigating/modelling:
○ Mount forces
- Excitation of driveline with full cyclic torque
- Cranking (start-up) and engine warm up
- Detailed friction modelling

Methods for predicting the engine torque are based on the widely used Wiebe function. Where more detailed combustion or heat release models are re- 
quired, bespoke models can be "plugged in" provided their interfaces are compatible with the Engine Library architecture.

\subsection{Surrogate models and real time simulation}

To minimise CPU time for the simulations and achieve real-time simulation, in addition to a conventional multi-cylinder architecture, the Engines library adopts an option presented in [3]. A singlecylinder model is parametised and a duplicating model replicates the variables of interest, namely the flows, temperatures and torque generated. With appropriate connections made from the variable duplicating model to the relevant components, a multi cylinder engine model can be simulated with minimised CPU time and negligible loss of accuracy $(<2 \%)$. This solution has been successfully implemented in both variants of the Engines library

The surrogate model method also allows a reduction in architecture diagram complexity and a quick and effective way to vary the number of cylinders in the engine.

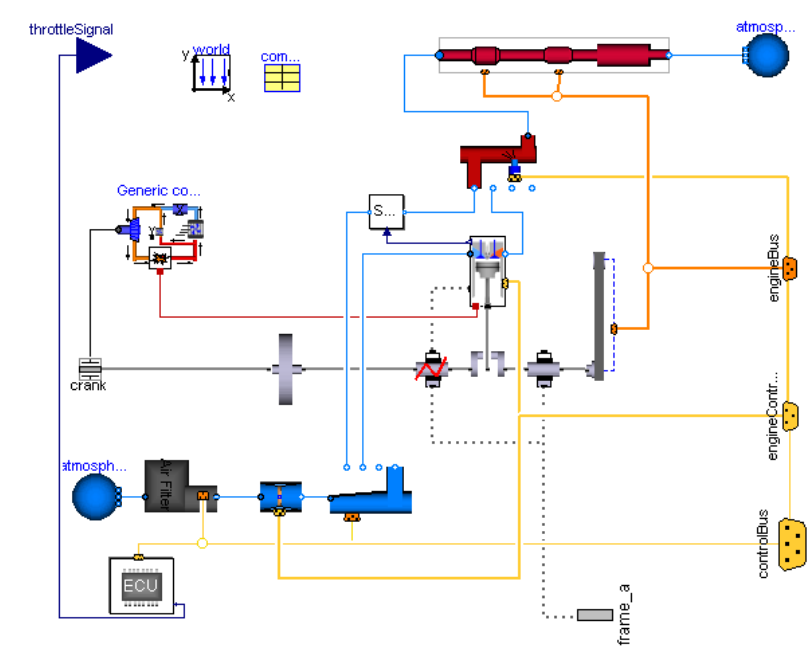

Example of surrogate MVEM layout

\subsection{Thermal effects}

Pipe wall and fluid thermal effects are taken care of using the Modelica Fluid dynamic pipe models (Modelica.Fluid.Pipes.DynamicPipe) [1]. In addition to the heat transfer models within Modelica.Fluid, the Engines library includes a further heat transfer model where existing or bespoke Nusselt Number correlations can be "plugged in" with a drop-down list of options [4].

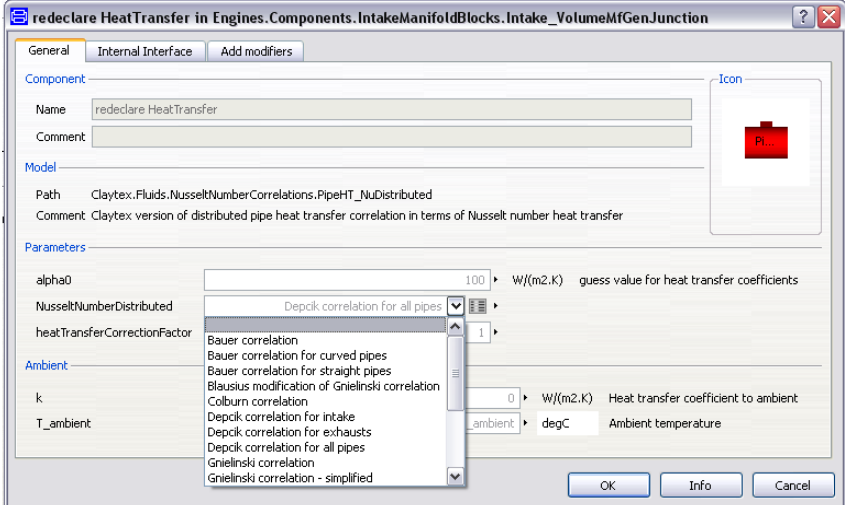

Heat transfer GUI with Nusselt number correlation dropdown list

These correlations make use of the Re (Reynolds number), Pr (Prandtl number), the medium temperature and the medium pressure and are used in the equations below to calculate the heat transfer between the fluid and the pipe wall.

A heat transfer correction factor has been introduced for correlation purposes.

\section{Q_flows $=\{$ alphas $[\mathrm{i}] *$ surfaceAreas[i]*(heatPorts[i].T - Ts[i])*nParallel for $\mathrm{i}$ in 1:n $\}$; \\ alphas $=$ lambdas $*$ heatTransferCorrectionFactor.$*$ Nus ./ dimensions;}

where for the descretised pipe:

- Q_flows is the vector of heat flows

- alphas is the vector of heat transfer coefficients

- $\quad$ surfaceAreas is the vector of heat transfer surface areas

- heatPorts.T and Ts are the vectors of the heat port temperatures and fluid temperatures

- nParallel is the number of parallel pipes

- lambdas is the vector of thermal conductivities

- Nus is the vector of Nusselt numbers

- Dimensions is the vector of pipe diameters

\section{Pipe Wall Heat Dissipation Models}

Three options for pipe wall heat transfer model are available within the Engines library models. The first is a fixed temperature model where the user fixes the pipe wall temperatures for steady state tests. The second option models the thermal energy dissipation to ambient (including convection and radiation ef- 
fects). This might be of particular interest when performing transient engine tests where the temperatures of the pipe walls throughout the test may vary significantly.

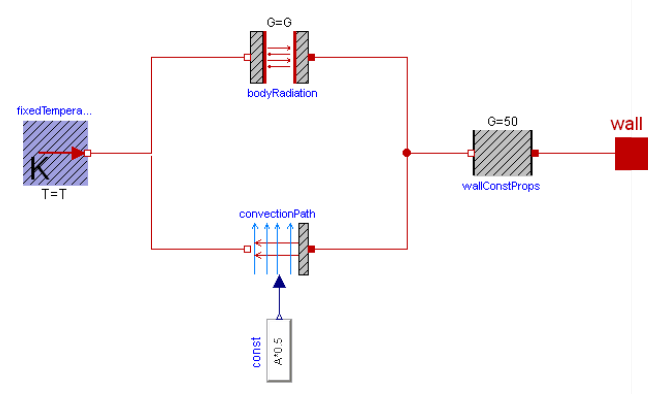

Heat transfer-to-air model

The third is a bespoke model for catalytic converter heat transfer. This model accounts for the thermal capacities of the catalyst brick and the casing of the catalytic converter. It can be used to model the catalyst light off in a transient test.

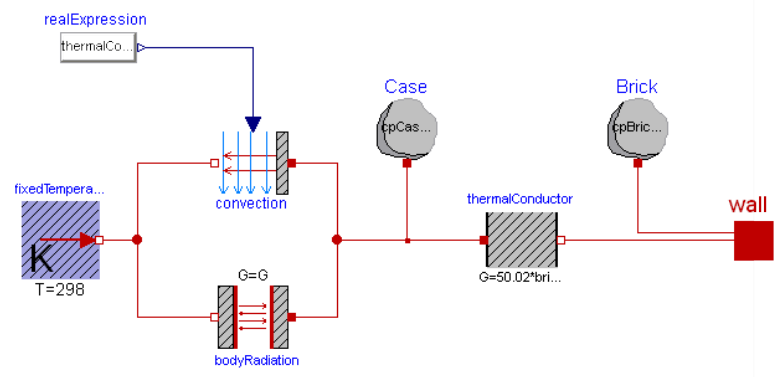

Catalyst heat dissipation model

All models are based on Modelica.Thermal library components.

\subsection{Engine Friction}

Bearing models with friction take into account the crank shaft, camshaft support and valvetrain mechanism bearing friction. Established friction models have been implemented [5] [6] [7] [8] which describe the friction torque resulting from hydrodynamic and rolling contact bearings. A more detailed description of the bearing models can be found in section 3 .

\section{Piston Assembly}

Piston assembly friction is cyclic and related to the cylinder pressure, piston speed and piston ring geometry. Piston skirt - cylinder liner friction is also modelled and relates to piston speed, geometry and the resultant lateral forces. Boundary and Hydrodynamic friction types are modelled [7] [8].

\section{Valve train}

Cam friction is also cyclic and calculated with reference to the cyclic vertical and horizontal loading, cam geometry and material.

The sliding friction of the cams has been modelled as a multi-stage solution [5]. The type of lubrication existing at the cam sliding surface is determined by means of the equation below where $\lambda$ is the film thickness parameter, $\mathrm{H}$ is the minimum film thickness for hydrodynamic lubrication, Rx is the effective radius of the sliding pair of surfaces, and $\sigma$ is the measured composite surface roughness of the two surfaces [5].

$$
\lambda=\frac{\mathrm{H}^{*} \mathrm{Rx}}{\sigma}
$$

$\lambda>1$ denotes a hydrodynamic lubrication regime

$\lambda \cong 0$ denotes a boundary lubrication regime and

$0>\lambda>1$ denotes a mixed lubrication regime

Both viscous and boundary components of the sliding surface friction are then calculated and summed to yield the total sliding surface friction.

$$
F_{t o t}=F_{b}+F_{v}
$$

\subsection{Aftertreatment}

\section{Catalysis}

3-way catalytic converter models make use of the species tracking within the exhaust medium to model the catalysis of the pollutant emissions.

The catalysis is modelled phenomenologically using efficiency maps. The catalysis efficiency is dependant on the air fuel ratio and the brick temperatures. Each of the pollutant emissions is reduced accordingly and adjustments are made to the mass fractions of other species within the medium model [9] [10] [11].

The heat release is calculated according to the number of moles of each pollutant emission that have been converted. In addition, a Secondary Air Injection and Diesel particulate filter have been developed. 


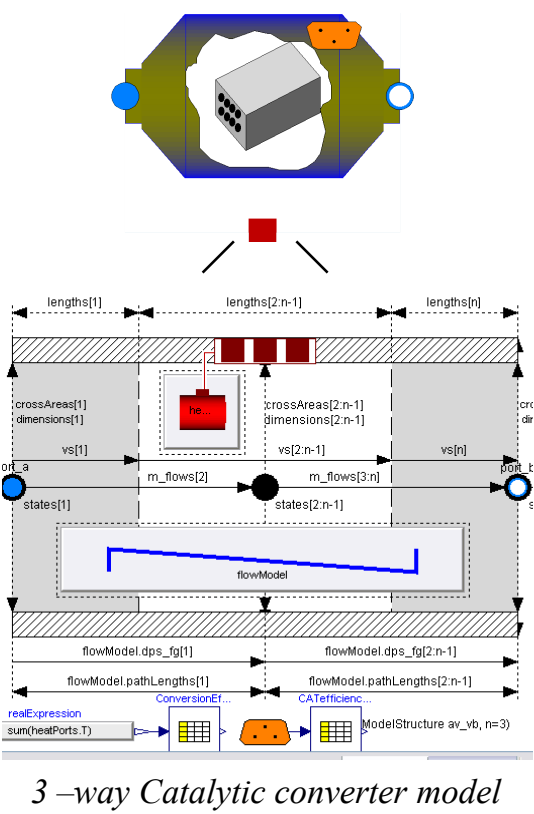

\section{Engine Control Unit}

A generic engine management system is provided for basic control of the throttle, variable valve timing, emissions control systems (such as Exhaust Gas Recirculation and Secondary Air Injection), pressure charging, spark timing and injection timing and duration. All the required engine sensor signals are available to the ECU via the control bus to ensure interchangeability with a bespoke control system. A template using this control bus is available for such systems to be developed.

\section{Powertrain Dynamics Library}

The PTDynamics library is a new library for modelling rotating MultiBody systems. The components are designed to be a more efficient way to model rotational mechanics capturing the full MultiBody effects of the rotation. The development was driven from the fact the 1D Rotational library is too simple and the MultiBody library too inefficient for modelling transmission and driveline system dynamics.

PTDynamics includes shafts, bearings, gear mesh models, flexible joints and complex assemblies such as epicyclic and differential models along with the associated mounting systems.

\subsection{Rotational3D Approach}

Both the Engines and PTDynamics library make use of a new Rotaional3D library that uses the standard Modelica connector called FlangeWithBearing [12]. Within the scope of this library it is assumed that the
bearingFrame is always included and it is used to capture the MultiBody dynamics of the rotating system. Within the connector, the flange connector is used to capture the rotation angle of the body and the torque being applied around the axis of rotation. The bearingFrame is used to track the position, orientation, forces and other torques being applied to the body. The rotation of the body is assumed to always be about the local $\mathrm{x}$-axis of the bearingFrame.

To capture the full MultiBody dynamics of the body the rotation angle of the flange connector and the position and orientation of the bearingFrame connector have to be combined. The angular velocity of the flange connector and the bearingFrame connector are resolved in to a virtual orientation frame. The virtual orientation frame follows the bearingFrame orientation and rotates with the flange connector.

$\omega_{\text {body }}=\left\{\begin{array}{c}\omega_{\text {frame }}[1]+\omega_{\text {flange }} \\ \omega_{\text {frame }}[2] * \cos \left(\varphi_{\text {flange }}\right)+\omega_{\text {frame }}[3] * \sin \left(\varphi_{\text {flange }}\right) \\ \omega_{\text {frame }}[2] * \sin \left(\varphi_{\text {flange }}\right)+\omega_{\text {frame }}[3] * \cos \left(\varphi_{\text {flange }}\right)\end{array}\right\}$

Where $\varphi_{\text {flange }}, \omega_{\text {flange }}$ mean the angle and angular velocity of the flange connector, $\omega_{\text {frame }}$ means the angular velocity of the bearingFrame connector and $\omega_{\text {body }}$ is the overall angular velocity of the body resolved in to the virtual orientation frame.

From this the acceleration of the centre of mass can be determined and thus the inertial effects can be calculated to determine the forces acting on the bearingFrame due to the rotation on the body.

The torque acting in the flange $\left(\tau_{\text {flange }}\right)$ is the torque required to accelerate the rotation of the body and is dependent only on the inertia of the body around the principle axis of rotation. The reaction torque in to the bearingFrame is the difference between the torque in the flange connector and total torque acting on the body:

$$
\begin{aligned}
& \tau_{\text {flange }}=I_{x x} * \alpha_{\text {flange }} \\
& \tau_{\text {body }}=I * \alpha_{\text {body }}+\left(\omega_{\text {body }} \times\left(I * \omega_{\text {body }}\right)\right) \\
& \tau_{\text {frame }}=\tau_{\text {body,frame }}-\left\{\tau_{\text {flange }}, 0,0\right\}
\end{aligned}
$$

Where $\alpha_{\text {flange }}$ is the angular acceleration of the flange connector, $\mathrm{I}$ is the inertia matrix, $\alpha_{\mathrm{body}}$ is the angular acceleration of the body and $\tau_{\text {body }}$ is the torque acting on the body resolved into the virtual orientation frame, $\tau_{\text {body,frame }}$ is this torque resolved in to the orientation of the bearingFrame and $\tau_{\text {frame }}$ is the torque in the bearingFrame. 


\section{Benchmarking:}

To understand the benefits of this approach and to validate the method, a series of benchmark cases were developed to compare the Modelica 1D Rotational and MultiBody libraries with the new Rotational3D model. The simplest test case is shown below where a torque actuator is used to accelerate an inertia.

Using Dymola these test cases are reduced to the system of equations shown below. The 1D Rotational model is of course the most simple system possible but it ignores many important effects. The Rotational3D and MultiBody models both capture exactly the same effects and predict the same motion of the body and reaction forces and torques in to the world object.

The advantage of the Rotational3D approach is that the linear set of equations seen in the MultiBody example is eliminated and the number of time varying variables is reduced to 15 from 27.

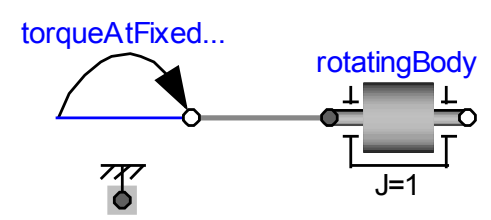

Continuous time states: 2 scalars

Time-varying variables: 2 scalars

Sizes of linear systems of equations: \{\}

Sizes after manipulation of the linear systems: \{\}

Sizes of nonlinear systems of equations: \{\}

Sizes after manipulation of the nonlinear systems: \{\}

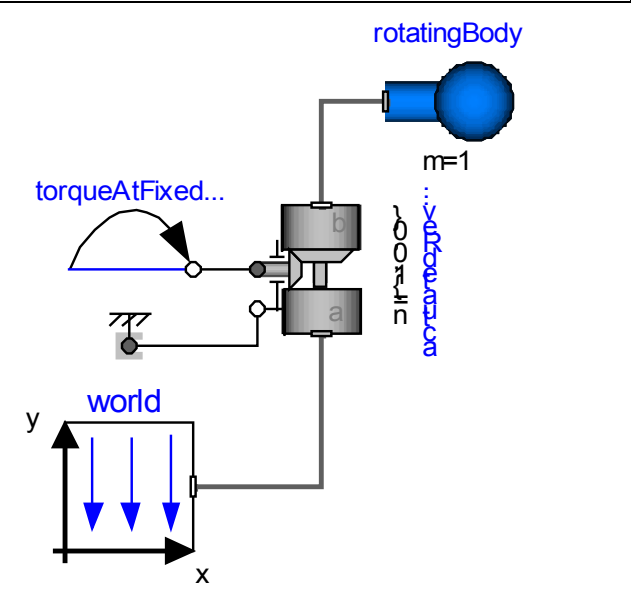

Continuous time states: 2 scalars

Time-varying variables: 27 scalars

Sizes of linear systems of equations: $\{3\}$

Sizes after manipulation of the linear systems: $\{0\}$

Sizes of nonlinear systems of equations: \{\}

Sizes after manipulation of the nonlinear systems: \{\}

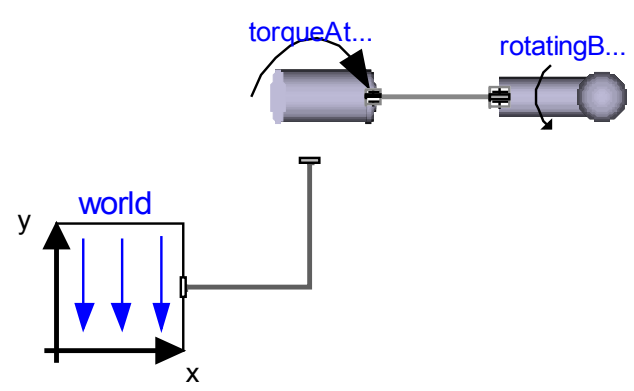

Continuous time states: 2 scalars

Time-varying variables: 15 scalars

Sizes of linear systems of equations: \{\}

Sizes after manipulation of the linear systems: \{\}

Sizes of nonlinear systems of equations: \{\}

Sizes after manipulation of the nonlinear systems: \{\}

\subsection{Bearings}

Bearings can be modelled as an ideal bearing which pins the shaft in position or with compliance that allows the shaft to move within the bearing. A number of friction models are available ranging from plain bearings to hydrodynamic lubrication so that different types of bearing can be modelled. Within each type of friction model we can define particular characteristics pertinent to that friction type.

In all bearings a full hydrodynamic model is available which makes use of the Sommerfeld number [7] [8] and bearing clearances to determine the lubrication regime. The model also accounts for the non-hydrodynamic type of lubrication under critical speeds and loads.

Rolling element bearing friction is also modelled with a selection of predefined friction coefficients that depend on the type of bearing and geometry of the rolling elements (single or double row ball, roller, taper roller).

Seal friction is taken into account using the normal force generated by the seal on the shaft and the defined coefficient of friction [13].

The rolling element bearing friction torque is calculated using the following formula:

$$
M_{r}=\left(F * f * \frac{d}{2}\right)+\left(F_{s} * f_{s} * \frac{d}{2}\right)
$$

$\mathrm{M}_{\mathrm{r}}=$ Friction torque $(\mathrm{Nm})$

$\mathrm{F}=$ Radial (or axial load) $(\mathrm{N})$

$\mathrm{F}_{\mathrm{s}}=$ Seal radial (or axial load) $(\mathrm{N})$

$\mathrm{f}=$ coefficient of friction of rolling bearing

$\mathrm{f}_{\mathrm{s}}=$ coefficient of friction of seal

$\mathrm{d}=$ Diameter of the bore of the bearing (Shaft diameter)

(m)

$\mathrm{D}=$ Outside diameter of the bearing $(\mathrm{m})$ 
Careful attention has to be paid by the modeller to avoid mechanical loops as these can be easily introduced. A mechanical loop is one where a position could be calculated via two or more paths and this cannot be handled automatically in Modelica. A simple case is illustrated below where the fixedTranslations form a loop.

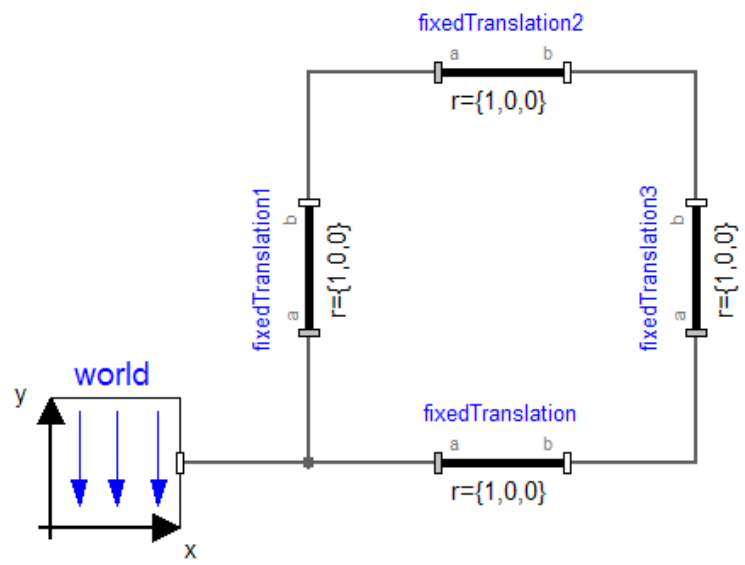

Using the shaft and bearing components within the PTDynamics library it would be very easy to create a mechanical loop. In the simplest case this would consist of a shaft with a support bearing at each end which would form a loop. To overcome this problem the bearing components include a flag breakMechanicalLoop and the modeller then has to follow the simple rule that only 1 bearing supporting a shaft can have this flag set to false, all the other bearings must have this flag set to true. The bearing icon is changed to reflect the value of this flag to make it easy for the modeller to verify this rule.

\subsection{Gears}

Within the PTDynamics library the fact that two gears are meshing is defined by adding a gear mesh component between the two gear bodies. This gear mesh model then calculates the forces and torques acting between the gears based on their relative positions and geometry.

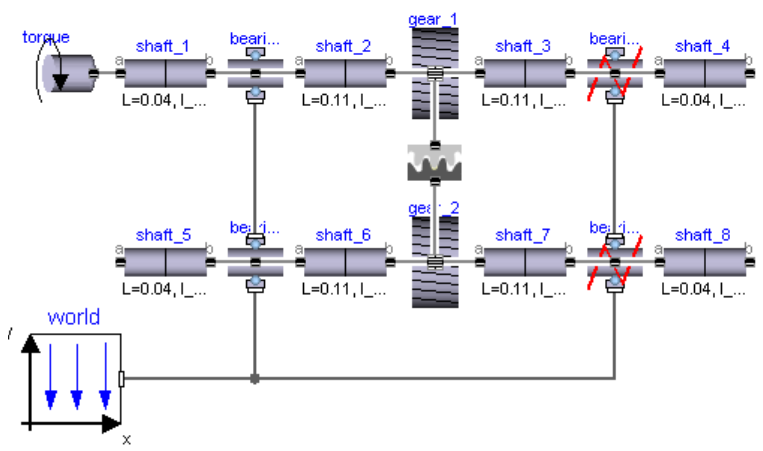

The mesh models account for the pressure angle and helix angle to calculate the radial and axial forces acting on the shafts. The mesh models also account for the rotation induced in the shafts due to their axial movement and the sliding of the gear teeth against one another in non-spur gears. A range of different mesh models will be available to account for mesh stiffness and backlash within the gear pair.

Specialised mesh models are also defined for use in epicyclic and differential gears that allow the forces and torques acting on each gear to be calculated.

\subsection{Joints}

A range of different shaft couplings are available allowing articulation of the shafts. The Joints can all include torsional compliance effects such as backlash and account for the cyclic speed and torque effects present in joints such as the Hookes joint (Universal joint). Also available are plunging joints with friction and constant velocity joints.

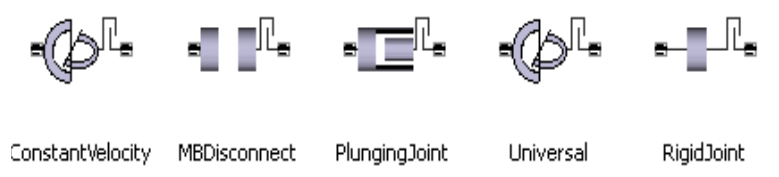

To simplify models and eliminate joint articulation two special joints are provided, a rigid joint and one called a MBDisconnect joint. The rigid joint eliminates all degrees of freedom in the joint. The MBDisconnect joint provides a complete break in the MultiBody system.

\section{Coupling the Engines and Power- Train Dynamics Libraries}

Being able to replicate engine torque pulsations through the driveline for torsional vibration analysis is a key part of driveline design. The designer could just input a table based torque signal obtained by means of a logged test but the ability to dial in a prescribed throttle profile and being able to vary it for different tests, thus being able to generate the corresponding torque is a desirable feature.

Using the VehicleInterfaces library [12] a complete vehicle model has been developed using the Engine library and the PTDynamics library to model the Transmission, Driveline and Chassis systems.

A rear-wheel drive vehicle has been developed and used to perform driveability tests whilst exploring the mount reaction forces and driveline vibration. 


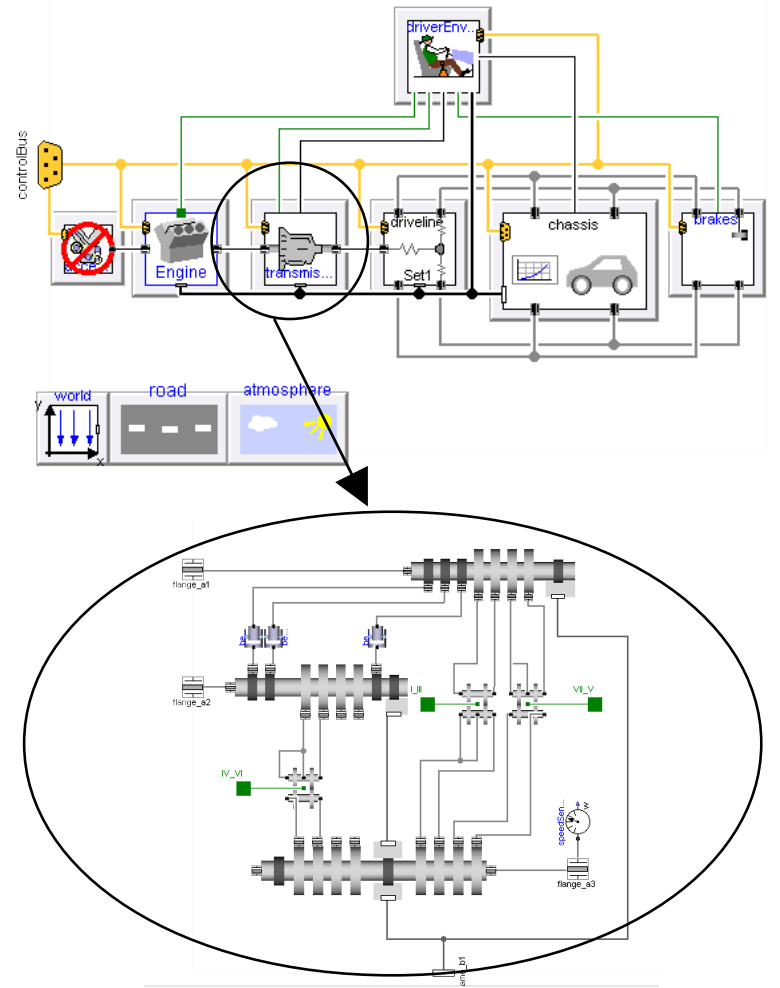

Diagram of the 6-speed dual clutch gearbox used in the vehicle model.

\section{Driveability study}

In this example, a $1.8 \mathrm{~L}$ gasoline inline 4-cylinder engine is coupled to a manual transmission, $1100 \mathrm{~kg}$ kerb weight, rear wheel drive vehicle and made to perform a typical tip-in/tip-out test whilst travelling in a straight line. The resulting driveline vibrations are displayed. The differential movement on its mounts is also shown during the tests

\subsection{The Engine}

The engine has been modelled using both variants of the library. For use during the tip-in/tip-out test the MVEL variant of the engine is utilised. The CAREL variant is used for correlation purposes and in other tests using the same vehicle.

The engine is a 4 cylinder inline, spark ignition, direct injection engine with a total volumetric displacement of $1800 \mathrm{cc}$. It's naturally aspirated with 4 valves per cylinder and direct acting camshafts. The engine is mounted in the vehicle with 3 non-linear mounts.

\subsection{The transmission}

The vehicle modelled uses a 6-speed dual clutch transmission rigidly mounted to the engine.
The model includes backlash, synchro parallel gear mesh models and compliant bearing models with relevant parasitic loss (friction) and efficiency models.

\subsection{The driveline}

An open differential with 4-point mounting system has been used. The PTDynamics Differential Gear and Bevel Gear mesh models have been used for both differential assembly and the pinion-wheel mesh. Parasitic losses have been implemented within the bearings and the power-dependant losses are modelled as efficiency terms within the mesh models. All main shafts and joints are compliant with backlash applied to specific joints within the driveline model.

\subsection{The Chassis}

A Pacejka Magic Formula tyre model was used, utilising the SAE J2452 rolling resistance model. The vehicle body used a 3 degree of freedom model capturing pitch and bounce in addition to the longitudinal motion of the vehicle.

\subsection{Correlation}

Vehicle coast downs were performed for the vehicle in each gear to correlate the aerodynamic, rolling resistance and driveline losses.

\subsection{Tip-in tests}

The terms tip-in and tip-out are used within driveability tests to describe a positive and negative step change in throttle input. In the context of this paper, the tip-in will refer to a $70 \%$ step throttle opening. Tip-out will refer to a full throttle pedal lift off.

Tip-in tests were performed using open-loop control of the driver throttle pedal. The same test can be repeated in each of the gears in the transmission and for varying amounts of throttle opening applied.

The vehicle is left to settle to a predefined speed at which a tip in event is triggered. At a predefined engine speed we then trigger the tip-out event.

The oscillatory phenomena at the investigated events will be captured for driveline vibration analysis.

\section{Results}

\subsection{Engine: MVEM and CAREM vs Test Data}

Simulation data from a fully correlated engine model generated in a widely used engine simulation pack- 
age was used to correlate both a MVEM (Mean Value Engine Model) and a CAREM (Crank Angle Resolved Engine Model).

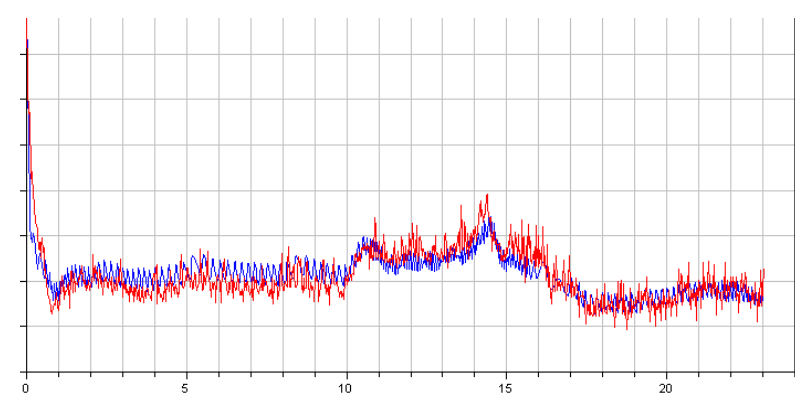

CAREM results (blue) vs. correlated engine model (red)

\section{Real Time with MVEM}

Simulation of the first $23 \mathrm{~s}$ of the ECE15 cycle are completed within $11 \mathrm{~s}$ of CPU time. The model includes the catalysis of the exhaust gasses and the modelling of the 3-way catalytic converter brick light-off.

\section{MVEM VS. CAREM}
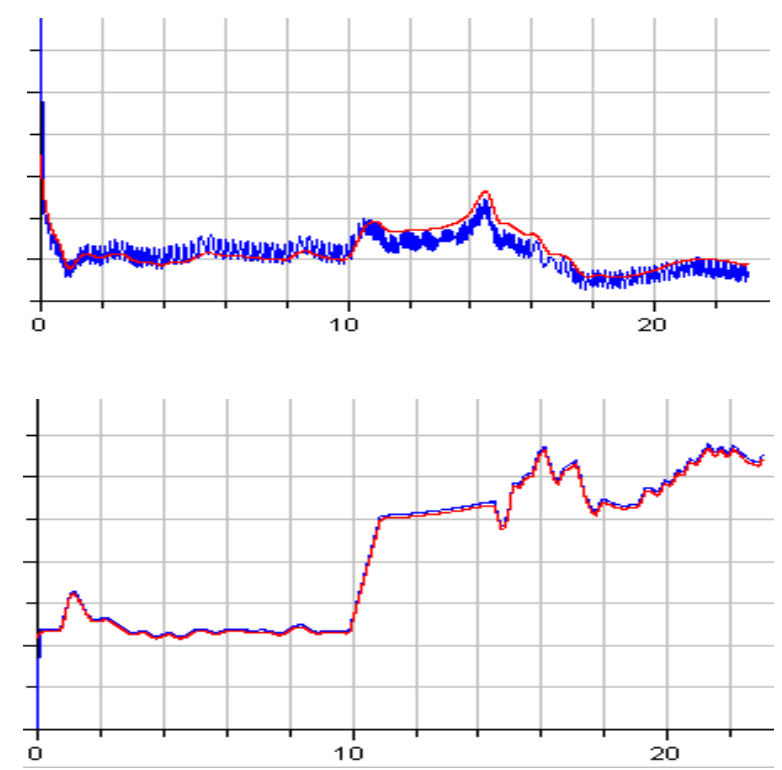

Plot of plenum pressure and throttle body mass flow rate for a CAREM (blue) and MVEM (red).

The results from the MVEM and CAREM were compared to each other to ascertain similarity within the two models.

The plenum pressure error comparing a MVEM and CAREM representation of the same engine is within $+/-5 \%$. The throttle body mass flow rate is within $+/-1 \%$. The discrepancy during the first 10 seconds of the plenum pressure plot can be attributed to slight a miscalibration of the MVEM mass flow rate function at low throttle openings and engine speeds.

\subsection{Multi Cylinder vs. Surrogate}

The advantages of surrogate models lie in model simplification, which translates to a reduction in CPU time. A test was performed to demonstrate negligible loss in accuracy when using the surrogate cylinder engine model to replace a multi-cylinder engine model.

Whilst the error was contained within $+/-2 \%$, the benefit in running a surrogate CAREM model over a multi-cylinder CAREM is a 4.5 times reduction in CPU time.

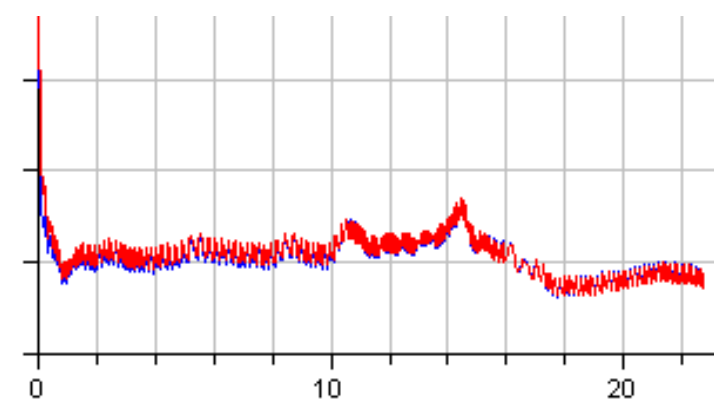

Plot of plenum pressure for a CAREM multi-cylinder representation (blue) vs. a surrogate representation of the same 4-cylinder SI engine (red).

\subsection{Tip-in tip-out tests}

Results from a second gear tip-in tip-out test are shown below.

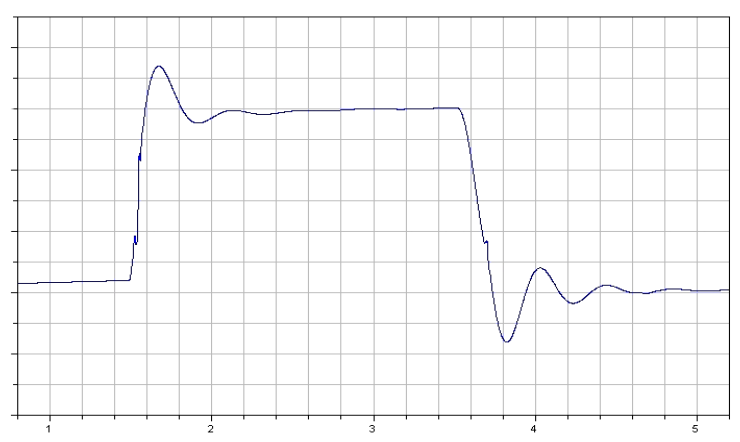

Vehicle longitudinal acceleration
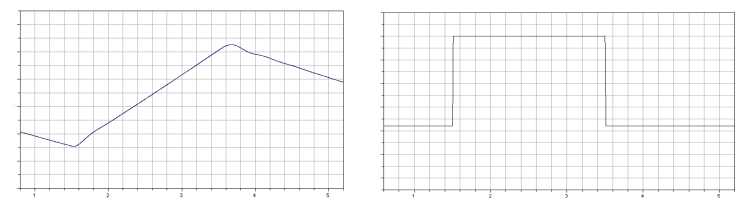

Vehicle longitudinal velocity and engine throttle position 
The vehicle longitudinal acceleration presents damped oscillations typical of this type of manoeuvre at and after the tip-in and tip-out events. The backlash in the driveline is particularly visible between the tip in/out events and the first peak in the vehicle acceleration oscillations as shown below.

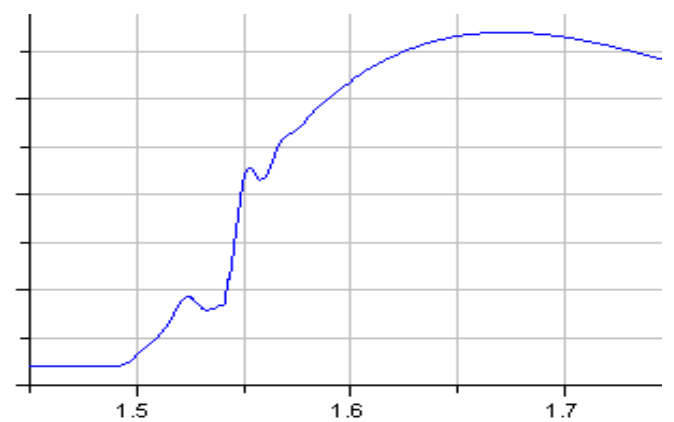

Vehicle longitudinal acceleration showing discontinuities due to backlash regions being crossed.

The mount displacements for the differential were in agreement with expectations for this type of manoeuvre.

The relative roll of the differential is visible shortly after the tip-in and tip-out points (1.5s and $3.5 \mathrm{~s})$. A positive pitch angle for the differential assembly is demonstrated during the tip-in acceleration event (front mounts have moved upwards and rear mounts have moved downwards) whilst a negative one is shown for deceleration event.

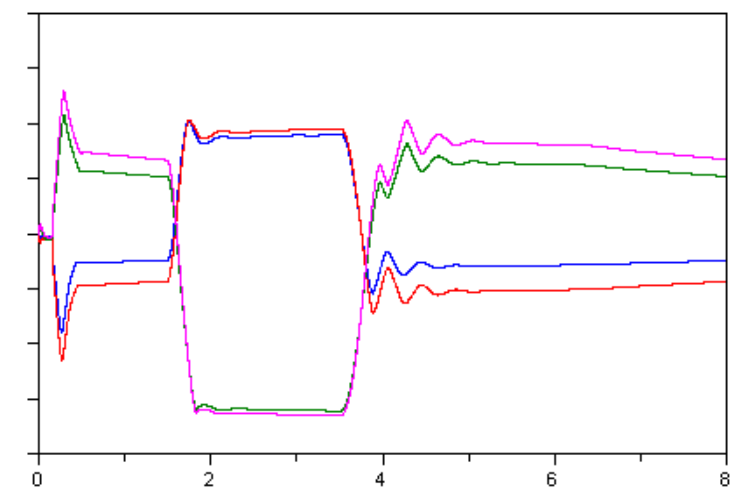

Vertical displacement of the 4 differential mounts during the tip-in/tip-out test. The blue and red lines represent the front mounts and the green and magenta lines represent the rear mounts.

\section{Conclusions}

Two new libraries have been developed for modelling Engines and Powertrain system dynamics. These both utilise a new approach to modelling the mechanics that capture the full MultiBody effects in a more efficient manner than the standard Modelica MultiBody library.

Using these two libraries a complete vehicle model has been built to study a range of different behaviours. Results for the engine model operation on its own are presented along with results from a tipin/tip-out manoeuvre.

The developed vehicle model can therefore be used for driveline vibration analysis as well as performance and vehicle dynamics tests. By plotting the mode shapes we can identify the sources of vibration and adjust the source components accordingly to counteract undesired phenomena.

\section{References}

1. Franke, R. et al. "Standardization of thermofluid modeling in Modelica_Fluid 1.0". Modelica Conference 2009.

2. Hendricks et al. "Modelling of the Intake Manifold Filling Dynamics" SAE 960037 1996.

3. John J. Batteh Charles E. Newman. "Detailed Simulation of Turbocharged Engines with Modelica" Modelica Conference, 2008.

4. Finol C A and Robinson K. "Thermal modeling of modern engines: a review of empirical correlations to estimate the in-cylinder heat transfer coefficient". Department of Mechanical Engineering, University of Bath, UK.

5. Yang et al. "A Valve Train Friction and Lubrication Analysis Model and Its Application in a Cam/Tappet Wear Study" SAE 962030 1996.

6. R. C. Coy. "Practical applications of lubrication models in engines" Tribology International vol. 31 No. 10.

7. Heywood J.B. "Internal Combustion Engine Fundamentals" McGraw Hill.

8. Stone, R. "Introduction to Internal Combustion Engines" SAE International, 1999

9. Masoudi. M. "Pressure Drop of Segmented Diesel Particulate Filters". SAE 2005-0109712005

10. Kladopoulou et al. "A study Describing the Performance of Diesel Particulate Filters During Loading and Regeneration - A lumped Parameter Model for Control Applications". SAE 2003-01-0842 2003

11. Silva et al. "Evaluation of SI engine exhaust gas emission upstream and downstream of the 
catalytic converter". Mechanical Engineering Department, Technical University of Lisbon, Portugal. 2006

12. Dempsey, M. et al. "Coordinated automotive libraries for vehicle system modeling”. Modelica Conference 2006.

13. www.roymech.co.uk

14. Kandylas I. P. and Stamatelos A.M. "Engine exhaust system design based on heat transfer computation" Energy Conversion and Management 40 (1999).

15. Batteh J. J. and Kenny P. J. "Modelling the Dynamics of Vehicle Fuel Systems". Modelica Conference 2006. 